\title{
Recurrent major depression, ataxia, and cardiomyopathy: association with a novel POLG mutation?
}

This article was published in the following Dove Press journal:

Neuropsychiatric Disease and Treatment

I3 May 201 I

Number of times this article has been viewed

\author{
Willem MA Verhoeven ${ }^{1,2}$ \\ Jos IM Egger ${ }^{1,3,4}$ \\ Berry PH Kremer \\ Boudewijn JHB de Pont ${ }^{1}$ \\ Carlo LM Marcelis ${ }^{6}$ \\ 'Vincent van Gogh Institute for \\ Psychiatry, Centre of Excellence \\ for Neuropsychiatry, Venray, The \\ Netherlands; ${ }^{2}$ Erasmus University \\ Medical Centre, Department \\ of Psychiatry, Rotterdam, The \\ Netherlands; ${ }^{3}$ Behavioural Science \\ Institute, Radboud University \\ Nijmegen, Nijmegen, The Netherlands; \\ ${ }^{4}$ Donders Institute for Brain, \\ Cognition and Behaviour, Centre \\ for Cognition, Radboud University \\ Nijmegen, Nijmegen, The Netherlands; \\ ${ }^{5}$ Department of Neurology, University \\ Medical Centre Groningen, The \\ Netherlands; ${ }^{6}$ Department of Human \\ Genetics, Radboud University \\ Nijmegen Medical Centre, Nijmegen, \\ The Netherlands
}

Correspondence: Willem MA Verhoeven Vincent van Gogh Institute for Psychiatry, Stationsweg 46, 5803AC Venray, The Netherlands $\mathrm{Tel}+3$ I 478527339

Fax +3 I 478527।I0

Email wverhoeven@vvgi.nl
Abstract: At present, more than 100 disease mutations in mitochondrial DNA polymerase $\gamma$ $(P O L G)$ have been indentified that are causally related to an array of neuropsychiatric diseases affecting multiple systems. Both autosomal recessive and autosomal dominant forms can be delineated, the latter being associated with Parkinsonism and depressive or psychotic syndromes. In this report, a middle-aged female patient with recurrent major depression with melancholic features, slowly progressive gait instability, and dilated cardiomyopathy is described. Detailed diagnostic evaluation was performed to elucidate the supposed relationship between ataxia, cardiomyopathy, and major depression with melancholia. After extensive genetic and metabolic investigation, a nucleotide substitution c. $2207 \mathrm{~A} \rightarrow \mathrm{G}$ in the $P O L G$ gene resulting in amino acid change Asn 736Ser in exon 13 was demonstrated. This mutation was considered to be compatible with a mitochondrial disorder and implicated in the pathophysiology of the neuropsychiatric syndrome. It is concluded that this novel POLG mutation forms the most parsimonious etiological explanation for the here-described combination of ataxia, major depression, and cardiomyopathy. Therefore, in patients with a complex neuropsychiatric presentation, extensive diagnostic analysis is warranted, including the search for mitochondriopathies, in order to avoid unnecessary delay of adequate treatment.

Keywords: DNA polymerase $\gamma$, mitochondrial disease, cerebellar ataxia, major depression

\section{Introduction}

Cerebellar ataxias, of which the spinocerebellar ataxias (SCAs) are the most prevalent, represent a heterogeneous group of neurodegenerative disorders that, according to their genetic etiology, can be differentiated among autosomal dominant, autosomal recessive, X-linked, and maternally inherited syndromes. ${ }^{1,2}$ Over the last decade, novel mitochondrial genetic diseases have been identified in which mutations in DNA polymerase $\gamma$ (POLG [MIM 174763]) gene are involved. POLG1 is the only DNA polymerase in human mitochondria and is essential for mitochondrial (mt)DNA replication and repair. It has to be stressed that functional genetic variants of $P O L G$ are present in about 0.5 percent of the normal population. Defects in mtDNA replication lead to mitochondrial dysfunction and disease. Originally, primary mtDNA mutations were thought to be the major cause of human mitochondriopathies. ${ }^{3}$ Nowadays, however, a great number of novel diseases have been described in which POLG mutations are causative through a secondary effect on mtDNA. These syndromes are thought to have a common European ancestry and may manifest from early infancy to late middle age. ${ }^{4,5}$

To date, POLG mutations have been found to be causal in a great number of mitochondrial diseases with a very heterogeneous clinical manifestation and affecting 
multiple systems. ${ }^{6}$ In 2001, Van Goethem and coworkers identified a mutation in $P O L G$ in a Belgian pedigree with progressive external ophthalmoplegia. ${ }^{7}$ Thereafter, mutations in $P O L G$ were found in a great variety of diseases like Alpers syndrome, SCA negative cerebellar ataxia, and Charcot-Marie-Tooth disease..$^{8-10}$

POLG mutations cause an overlapping clinical spectrum of diseases with both dominant and recessive modes of inheritance. Autosomal recessive forms often present with external ophthalmoplegia, peripheral neuropathy, ataxia, and epilepsy. ${ }^{5,11}$ Autosomal dominant forms are mostly characterized by symptoms within the ataxia-neuropathy range and by progressive external ophthalmoplegia. In addition, these autosomal dominant syndromes are reported to be associated with an array of neuropsychiatric symptoms from the Parkinson spectrum and from the psychotic and depressive domains of which major depression has the highest incidence. ${ }^{12-14}$

In this report, a middle-aged female patient is described with a long history of recurrent depression and slowly progressive gait instability, as well as dilated cardiomyopathy for the 5-year period prior to admission. A descriptive diagnosis of progressive cerebellar ataxia with bilateral pyramidal syndrome and cardiomyopathy was made. Later, extensive genetic work-up demonstrated a $P O L G$ mutation which was thought to be of pathogenic significance.

\section{Clinical observations and results}

The patient is a 52-year-old single female born from nonconsanguineous parents. She has three healthy sisters. Her only brother died in his early fifties, allegedly from gastric hemorrhage. Her mother and father died aged 55 and 80 from acute cardiac arrest, most probably due to cardiomyopathy, and cerebral hemorrhagia respectively. There is no family history of mental disorders or hereditary diseases. At the age of 33 she gave birth to a healthy son who is mildly mentally retarded. The patient showed a normal developmental trajectory, completed primary school followed by domestic training, and was subsequently employed in nondemanding jobs. Aged 17, she was involved in a serious car accident resulting in cerebral concussion and various fractures of the right foot and knee for which she underwent several orthopedic surgical corrections. Since that time, the patient showed an unstable gait and balance, which with physical therapy gradually disappeared. For a period of 20 years, she was able to perform her elementary activities at a laundry, not hindered by motor impairments. At the age of 37 years, however, she was referred to a rehabilitation physician because she'd had slowly progressive gait instability for a period of 2 years.
Physical examination disclosed a mild ataxia that was interpreted as a result of the cerebral concussion. In addition, emotional and affective instability were noticed. No neuroradiological investigation was performed.

Over subsequent years, the patient showed recurrent depressive symptoms for which she was treated by her general practitioner with several antidepressants. Aged 44, she underwent surgery for bilateral subcapsular posterior cataract. At the same age, she was admitted to a psychiatric hospital because of domestic neglect, inactivity, paranoid ideation, and symptoms of major depression with melancholic features. Neurological examination demonstrated bilateral ataxia and a slightly dysarthric speech. CT (computed tomography)-scanning of the brain revealed a moderate cerebellar atrophy. The neurological symptoms were considered to be of posttraumatic origin. Treatment with clomipramine resulted in complete remission of depressive symptoms, and the patient was followed up at the outpatient department of psychiatry. At the age of 47 , she developed complaints of fatigue and shortness of breath for which she was referred to a cardiologist. A diagnosis of dilated cardiomyopathy with a cardiac output of less than $30 \%$ was made, and treatment with the beta blocker bisoprolol was started. Over the subsequent 6 years, several depressive episodes with psychotic phenomena and suicidal events occurred that were treated with various psychotropic agents. Because of serious impairment of general functioning and persistent depressive and psychotic symptoms, the patient was referred to the specialized department of neuropsychiatry for extensive diagnostic assessment.

At admission, extreme loss of energy and marked hypokinesia were prominent, and as a consequence, the patient was partially wheelchair bound. Since these phenomena were caused by her cardiomyopathy and use of antipsychotics respectively, treatment with bisoprolol was first optimalized and psychotropics were gradually tapered off. Thereafter, neuropsychiatric examination revealed a slight paranoid ideation and suicidal thoughts. There was significant loss of initiative, flattening of affect, depressed/dysphoric mood, and delusional ideas of nihilism, as well as anosognosia. Neuropsychological functioning was characterized by markedly lowered information processing and a relatively intact working memory. Her total IQ was 69 (WAIS-III ${ }^{15}$ ), indicative of below average intellectual functioning. The patient was friendly and cooperative but refused an extensive testing program. ECG-recording showed an incomplete left ventricular bundle branch block with inverted T-waves. Routine hematological and biochemical laboratory tests disclosed a 
mild dyslipidemia only (cholesterol $6.7 \mathrm{mmol} / \mathrm{L}$, with other lipid parameters normal).

Treatment with citalopram in a maximal dose of $20 \mathrm{mg}$ daily resulted in a gradual reduction of depressive symptoms, even though inactivity and loss of energy persisted and cerebellar dysfunctions became more prominent. An MRI (magnetic resonance imaging) scan of the brain demonstrated marked cerebellar atrophy without any supratentorial lesions (Figure 1). Karyotyping revealed no abnormalities.

Given the combination of ataxia and cardiomyopathy, the possibility of a hereditary spinocerebellar ataxia was considered, and hence an extensive neurological and etiological diagnostic procedure was started.

At neurological examination, the patient presented with slow speed and lack of initiative. Funduscopy revealed no abnormalities. There were saccadic intrusions of ocular smooth pursuit. Minimal bilateral thenar atrophia was noticed. No pareses were present. Speech was dysarthric and gait slightly ataxic but with normal base. All cerebellar tests were impaired. Sensibility was undisturbed in all modalities. Tendon reflexes of the arms and legs were hyperactive with bilateral positive Hoffmann-Trömner and Babinski signs. Snout reflex was present. An initial descriptive diagnosis

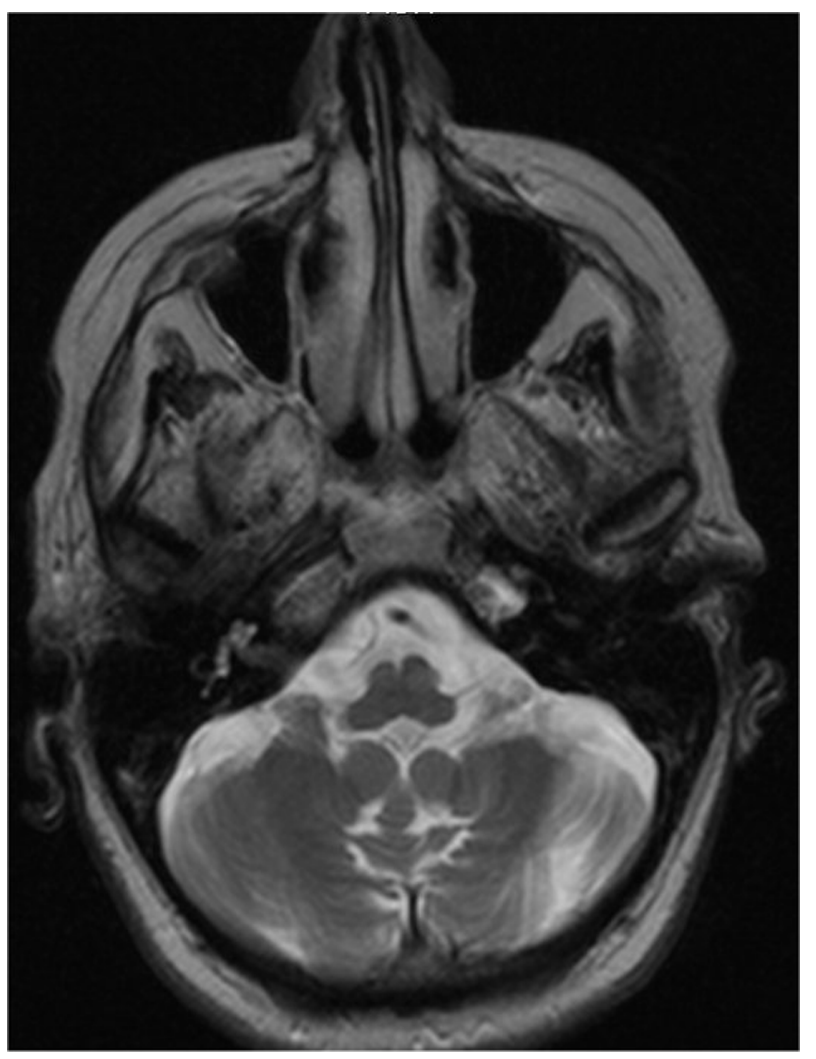

Figure I Axial, T2-weighted magnetic resonance image of the patient, showing atrophy of the cerebellum and the cerebellar vermis. of progressive cerebellar ataxia with bilateral pyramidal syndrome and cardiomyopathy, present for over a decade, was given. With mutation analysis, all known autosomal recessive cerebellar ataxias were excluded. Metabolic diseases like cerebrotendinous xanthomatosis and sphingolipidoses, especially Niemann-Pick disease type C, could not be demonstrated. The spectrum of amino acids in plasma as well as the levels of lactate and pyruvate were all normal. Mutation analysis of the POLG gene demonstrated a nucleotide substitution c.2207 $\mathrm{A} \rightarrow \mathrm{G}$ resulting in amino acid change Asn $736 \mathrm{Ser}$, which with sequence analysis appeared to be located in exon 13. This mutation was considered to be compatible with a mitochondrial disorder and assumed to be implicated in the pathophysiology of the neurological syndrome and the cardiomyopathy.

Thus, a provisional diagnosis of a mitochondrial $P O L G$ disease was made, probably an autosomal dominant heterozygotic form. Unfortunately, its clinical significance could not further be substantiated, since family members, in particular the son, were not available for additional testing. Although the depressive symptoms were no longer prominent, chronic hospitalization was necessary because of a slowly progressive increase of both cardiac dysfunction and neurological symptoms.

\section{Discussion}

The case described here is a typical example of a patient in whom an apparently common psychiatric disease, major depression, was initially diagnosed. Detailed recording of her medical life chart and family history, however, was indicative of the presence of a complex neurodegenerative disorder characterized by slowly progressive cerebellar ataxia and cardiomyopathy. Such a combination of symptoms goes along with a vast amount of differential diagnostic options, including hereditary ataxias and metabolic disorders (eg, lysosomal storage diseases, disturbances in amino acid metabolism, and cerebral cholesterosis). By means of extensive laboratory and genetic testing, all diagnostic hypotheses, other than a $P O L G$ mutation disease, could be falsified. In this patient, therefore, a tentative diagnosis of a POLG missense mutation was made.

Since there is an abundance of nucleotide substitutions that have been described in patients, Horvath and colleagues point at the diagnostic challenge that has to be mastered and provide a set of heuristic decision rules that delimit the clinical significance of a POLG mutation. ${ }^{16}$ The first principle states that the amino acid change is located in a functional relevant site of POLG. A second criterion is that that the 
mutation under study should be close to other established pathogenic $P O L G$ mutations. Given these principles and, in this case, the constellation of symptoms and the nucleotide change in the mutation c. $2207 \mathrm{~A} \rightarrow \mathrm{G}$, this novel mutation was considered to be pathogenically involved despite there being no information available on this mutation in normal populations.

It is well known that disorders within the mood and anxiety domain may be the initial sign of mitochondrial disorders. In fact, the prevalence in mitochondriopathies of a lifetime diagnosis of major depressive disorder is $54 \% .^{17}$ From several studies, it emerges that impairment of metabolism, especially at the mitochondrial level, may be involved in the pathophysiology of psychiatric diseases, including depressive disorders. ${ }^{18}$ Also POLG mutations have been reported to be associated with major depression. ${ }^{13}$ Therefore, major depression with melancholia as present in the current patient may be considered as part of the POLG-associated neuropsychiatric syndrome.

In conclusion, the presented case demonstrates a previously unreported POLG mutation accompanied by ataxia, cardiomyopathy, and major depression. Given the very heterogeneous phenotypical presentation of this multisystem disease, mutations involving the POLG gene should be considered in patients with major depressive disorder in whom a combination of unexplained neurological (eg, ataxia) and nonneurological (eg, cardiac dysfunction) is present.

\section{Acknowledgment}

This study is part of a collaborative project of the research group 'Psychopathology and Genetics'.

\section{Disclosure}

The authors report no conflicts of interest in this work.

\section{References}

1. Copeland WC. Inherited mitochondrial diseases of DNA replication. Ann Rev Med. 2008;59:131-146.

2. Finsterer J. Ataxias with autosomal, X-chromosomal or maternal inheritance. Can J Neurol Sci. 2009;36:409-428.

3. Finsterer J. Mitochondriopathies. Eur J Neurol. 2004;11:163-186.

4. Hakonen AH, Davidzon G, Dalemi R, et al. Abundance of the $P O L G$ disease mutations in Europe, Australia, New Zealand, and the United States explained by single ancient European founders. Eur J Hum Genet. 2007;15:779-783.

5. Hudson G, Chinnery PF. Mitochondrial DNA polymerase- $\gamma$ and human disease. Hum Mol Genet. 2006;15:R244-R252.

6. Hakonen AH, Heiskanen S, Juvonen V, et al. Am J Hum Genet. 2005; $77: 430-441$.

7. Van Goethem G, Dermaut B, Löfgren A, et al. Mutation of $P O L G$ is associated with progressive external ophthalmoplegia characterized by mtDNA deletions. Nat Genet. 2001;28:211-212.

8. Chan SSL, Copeland WC. DNA polymerase gamma and mitochondrial disease: understanding the consequences of $P O L G$ mutations. Biochim Biophys Acta. 2009;1787:312-319.

9. Blok MJ, van den Bosch BJ, Jongen E, et al. The unfolding clinical spectrum of POLG mutations. J Med Genet. 2009;46:776-785.

10. Wong LJC, Naviaux RK, Brunetti-Pierri N, et al. Molecular and clinical genetics of mitochondrial diseases due to POLG mutations. Hum Mutat. 2010;29:E150-E172.

11. Wintherthun S, Ferrari L, He L, et al. Autosomal recessive mitochondrial ataxic syndrome due to mitochondrial polymerase- $\gamma$ mutations. Neurology. 2005;64:1204-1208.

12. Luoma P, Melberg A, Rinne JO, et al. Parkinsonism, premature menopause, and mitochondrial DNA polymerase- $\gamma$ mutations: clinical and molecular genetic study. Lancet. 2004;364:875-882.

13. Koene S, Kozicz TL, Rodenburg RJT, et al. Major depression in adolescent children consecutively diagnosed with mitochondrial disorder. J Affect Disord. 2009;114:327-332.

14. Jou SH, Chiu NY, Liu CS. Mitochondrial dysfunction and psychiatric disorders. Chang Gung Med J. 2009;32:370-379.

15. Wechsler D. WAIS-III Technical Manual. San Antonio, TX: Psychological Corporation; 2002.

16. Horvath R, Hudson G, Ferrari G, et al. Phenotypic spectrum associated with mutations of the mitochondrial polymerase- $\gamma$ gene. Brain. 2006; 129:1674-1684

17. Fattal O, Link J, Quinn K, et al. Psychiatric comorbidity in 36 adults with mitochondrial cytopathies. CNS Spectr. 2007;12:429-438.

18. Rezin GT, Amboni G, Zugno AI, et al. Mitochondrial dysfunction and psychiatric disorders. Neurochem Res. 2009;34:1021-1029.
Neuropsychiatric Disease and Treatment

\section{Publish your work in this journal}

Neuropsychiatric Disease and Treatment is an international, peerreviewed journal of clinical therapeutics and pharmacology focusing on concise rapid reporting of clinical or pre-clinical studies on a range of neuropsychiatric and neurological disorders. This journal is indexed on PubMed Central, the 'PsycINFO' database and CAS, and is the official

\section{Dovepress}

journal of The International Neuropsychiatric Association (INA). The manuscript management system is completely online and includes a very quick and fair peer-review system, which is all easy to use. Visit $\mathrm{http}: / /$ www.dovepress.com/testimonials.php to read real quotes from published authors. 